\title{
PD-1/PD-L1 blockade in cancer treatment: perspectives and issues
}

\author{
Junzo Hamanishi $^{1} \cdot$ Masaki Mandai $^{2} \cdot$ Noriomi Matsumura $^{1} \cdot$ Kaoru Abiko $^{1}$ • \\ Tsukasa Baba ${ }^{1} \cdot$ Ikuo Konishi $^{1}$
}

Received: 27 January 2016 / Accepted: 31 January 2016 / Published online: 22 February 2016

(C) The Author(s) 2016. This article is published with open access at Springerlink.com

\begin{abstract}
Recent studies showed that tumor cells 'edit' host immunity in several ways to evade immune defenses in the tumor microenvironment. This phenomenon is called "cancer immune escape." One of the most important components in this system is an immunosuppressive co-signal (immune checkpoint) mediated by the PD-1 receptor and its ligand, PD-L1. PD-1 is mainly expressed on activated $\mathrm{T}$ cells, whereas PD-L1 is expressed on several types of tumor cells. Preclinical studies have shown that inhibition of the interaction between PD-1 and PD-L1 enhances the T-cell response and mediates antitumor activity. Several clinical trials of PD-1/PD-L1 signal-blockade agents have exhibited dramatic antitumor efficacy in patients with certain types of solid or hematological malignancies. In this review, we highlight recent clinical trials using anti-PD-1 or anti-PD-L1 antibodies against several types of malignancies, including a trial conducted in our department, and describe the clinical perspectives and issues regarding the PD-1/PD-L1 blockade in cancer treatment.
\end{abstract}

Keywords PD-1 - PD-L1 · Nivolumab - Immunotherapy · Biomarker · Value

Junzo Hamanishi

jnkhmns@kuhp.kyoto-u.ac.jp

1 Department of Gynecology and Obstetrics, Kyoto University Graduate School of Medicine, 54 Kawahara-cho, Shogoin, Sakyo-ku, Kyoto, Kyoto 606-8507, Japan

2 Department of Obstetrics and Gynecology, Kinki University Faculty of Medicine, Osaka, Japan

\section{Introduction}

Tumor cells have acquired several ways to escape from host immunity in the tumor microenvironment, called cancer immune escape via cancer immunoediting process [1]. During the past two decades, several studies of cancer immune escape revealed that one of the most important components of the underlying mechanism is an immunosuppressive co-signal (immune checkpoint) mediated by programmed cell death-1 (PD-1)/PD-1 ligand 1 (PD-L1) in the tumor microenvironment.

PD-1 was discovered by Tasuku Honjo and colleagues at Kyoto University in 1992 [2]. PD-1 expressed on T cells negatively regulates their antitumor effect [3-5]. PD-L1 engages with PD-1 to inhibit proliferation and cytokine production by $\mathrm{T}$ cells $[6,7]$. Several studies showed that under normal physiological conditions, PD-L1 is expressed in human tonsil, placental syncytiotrophoblast, monocyte, and lung, where it is involved in immune tolerance [8, 9]. Several preclinical reports also showed that inhibition of the interaction between PD-1 and PD-L1 enhances the T-cell response and mediates antitumor activity $[3-5,10]$. Additionally, PD-L1 is expressed on various human cancers, including urothelial cancers, gastrointestinal cancers, lung cancer, breast cancer, melanoma, and ovarian cancer, as well as on tumor-infiltrating immune cells in the tumor microenvironment $[8,9,11-18]$. Therefore, to provide proof of principle that the PD-1/PD-L1 blockade is effective against cancer, efforts were made to develop PD-1 inhibitors (anti-PD-1 or anti-PD-L1 antibody) for the treatment of human cancers (Fig. 1). The clinical trials performed to date have been highly successful.

In this review, we summarize recent clinical applications of PD-1/PD-L1 blockade in cancer treatment, as well as 
Fig. 1 Programmed death (PD)-1 inhibitors in cancer. PD-1 inhibitors (anti-PD-1 antibody and anti-PD-L1 antibody) block PD-1/PD-L1 signaling and induce anti-tumor immune reactivation at two checkpoints: cognitive phase (lymph node) and effector phase (tumor microenvironment). $\alpha P D-1 A b$ anti-PD-1 antibody, $\alpha P D-L 1 A b$ anti-PD-L1 antibody

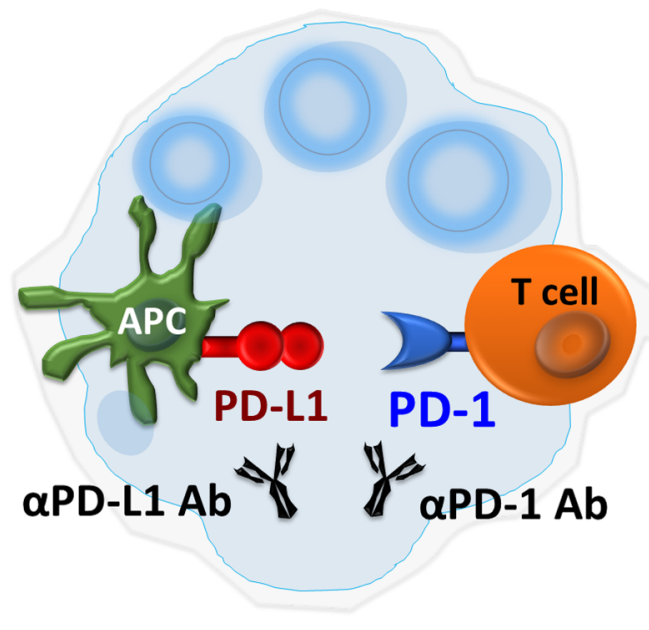

Lymph node $1^{\text {nd }}$ Checkpoint

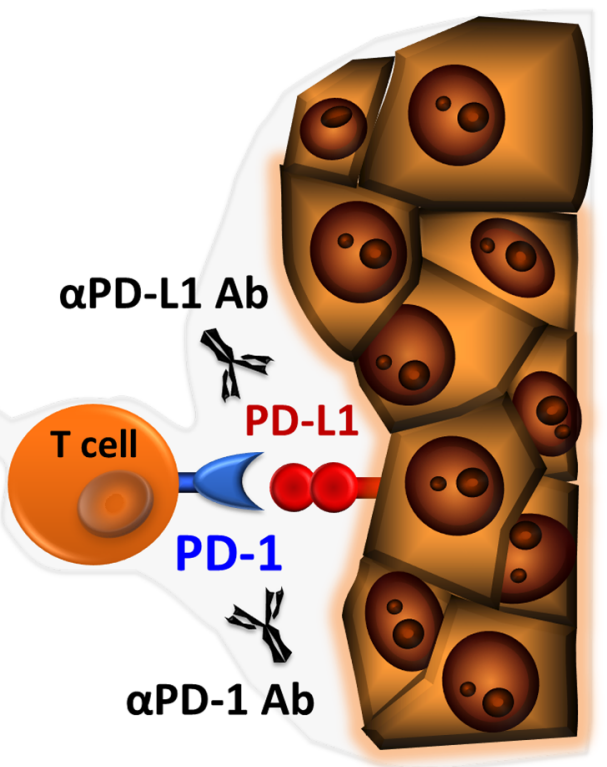

Cancer $2^{\text {st }}$ Checkpoint

Table 1 Programmed death (PD)-1 inhibitors (anti-PD-1 antibodies and anti-PD-L1 antibodies) in clinical testing

\begin{tabular}{|c|c|c|c|c|}
\hline Target & Agent & IgG class & Company & Approved \\
\hline \multirow[t]{5}{*}{ PD-1 } & Nivolumab (Opdivo ${ }^{\circledR}$, BMS-936558, MDX1106) & Human IgG4 & Bristol-Meyers Squibb/Ono & $\begin{array}{l}\text { Melanoma }^{1} \\
\text { Lung cancer }^{2}\end{array}$ \\
\hline & Pembrolizumab (Keytruda ${ }^{\circledR}$ MK-3475, lambrolizumab) & Humanized IgG4 & Merck & $\begin{array}{l}\text { Melanoma }^{3} \\
\text { Lung cancer }\end{array}$ \\
\hline & Pidilizumab (CT-011) & Humanized IgG1k & Cure Tech & \\
\hline & AMP-224 & PD-L2 IgG2a fusion protein & Amplimmune/GlaxoSmith Klein & \\
\hline & AMP-514 (MEDI0680) & PD-L2 fusion protein & Amplimmune/GlaxoSmith Klein & \\
\hline \multirow[t]{4}{*}{ PD-L1 } & BMS-936559 (MDX1105) & Human IgG4 & Bristol-Meyers Squibb & \\
\hline & Atezolizumab (MPDL3280A) & Human IgG1k & Roche/Genentech & \\
\hline & Durvalumab (MEDI4736) & Human IgG1k & MedImmune/AstraZeneca & \\
\hline & Avelumab (MSB0010718C) & Human IgG1 & Merck Serono/Pfizer & \\
\hline
\end{tabular}

1 Melanoma: approved in USA, EU, and Japan

2 Lung cancer: squamous cell lung cancer in USA an EU; non small lung cancer in Japan

3 Melanoma: approved in USA, EU

4 Lung cancer: PD-L1+ non small lung cancer in USA

discuss some pertinent perspectives and issues leading to further effective clinical application of PD-1 inhibitors to various malignant tumors in the near future.

\section{Clinical applications of PD-1 inhibitors in cancer}

In light of fundamental research, clinical studies using PD-1 pathway inhibitors against treatment-resistant solid tumors were initiated in the United States in 2006 [19].
To date, at least 200 such clinical studies have been carried out using nine types of antibody in at least 20 types of cancer, including both solid and hematological tumors; the total number of subjects worldwide is more than 20,000 (Table 1).

In 2010, the first phase I clinical trial of an anti-PD-1 antibody, nivolumab, was conducted in 39 patients with treatment-refractory solid tumors such as advanced melanoma, non-small cell lung cancer (NSCLC), renal cell carcinoma (RCC), prostate cancer, and colorectal 
cancer (CRC). The response rate (RR) was $7.7 \%$, including one durable complete response in CRC and two partial responses in melanoma and RCC. Only one serious adverse event (inflammatory colitis) was observed, so nivolumab was considered to be well tolerated [19].

Subsequently, in 2012, a phase I study of nivolumab was carried out in a total of 296 patients with NSCLC, melanoma, or RCC; the mean RR values were $18 \%, 28 \%$, and $27 \%$, respectively. The most frequent adverse effects (AEs) were rash, diarrhea, and itching (in $12 \%, 11 \%$, and $9 \%$ of subjects, respectively); AEs that occurred in $\geq 1 \%$ of subjects at grade 3 or 4 were diarrhea, hepatic dysfunction, and pneumonia. In particular, three subjects died of pneumonia; consequently, a cautionary note about immunological side reactions has been reported [20]. In addition, in a followup study, durable antitumor responses were observed, with overall survival (OS) of 9.9, 22.4, and 16.8 months for patients with NSCLC, RCC, and melanoma, respectively [21]. These studies constituted a turning point in the expansion of clinical applications of PD-1 inhibitors, and their results were reported in important publications that made major contributions to the ongoing rapid progress with these agents. Representative clinical trials in different types of cancer are summarized here.

\section{Melanoma}

Representative phase III clinical trials with anti-PD-1 antibodies, nivolumab and pembrolizumab, in patients with melanoma have led to the separate approval of both drugs. The first phase III trial with nivolumab as a firstline therapy was conducted in 418 treatment-free patients with metastatic melanoma lacking a mutation in BRAF. Patients were randomized to receive either nivolumab or chemotherapy with dacarbazine. The overall survival rate at 1 year was $73 \%$ for the nivolumab patients versus $42 \%$ in dacarbazine. Similarly, median progression-free survival was 5.1 months in the nivolumab group versus 2.2 months in the dacarbazine group. The objective response rate was $40 \%$ in the nivolumab group as compared with $14 \%$ in the dacarbazine group [22]. The second randomized phase III trial compared nivolumab to chemotherapy (dacarbazine or carboplatin/paclitaxel) in 405 patients with advanced metastatic melanoma [23]. In contrast to the previous trial, these patients had all been previously treated with ipilimumab, and a minority had also received a BRAF inhibitor. ORR was threefold higher in the nivolumab group than in the chemotherapy group (32\% vs. $11 \%$ ).

In another randomized phase III trial, 834 patients were with advanced melanoma were divided equally among three groups: pembrolizumab every 2 weeks, pembrolizumab every 3 weeks, or ipilimumab every 3 weeks. The estimated 6-month progression-free survival rates were $47 \%, 46.4 \%$, and $26.5 \%$, respectively, and the estimated overall 1-year survival rates were $74 \%, 68 \%$, and $58 \%$, respectively. The response rate was improved with pembrolizumab administered every 2 weeks $(33.7 \%)$ and every 3 weeks $(32.9 \%)$, as compared with ipilimumab (11.9\%) [24].

In 2014, the U.S. Food and Drug Administration (FDA) approved nivolumab and pembrolizumab for the treatment of advanced melanoma (both are limited to be approved for melanoma refractory to ipilimumab and with BRAF inhibitors, if the tumor harbors a BRAF mutation). Later, in 2015, the FDA additionally expanded an approval of nivolumab with BRAF wild-type and pembrolizumab for first treatment of unresectable or metastatic melanoma, each.

Similar to anti-PD-1 antibodies, anti-PD-L1 antibodies in phase I clinical trials for melanoma have shown promising antitumor activity. The first phase I trial of an antiPD-L1 antibody, BMS-936559, yielded a RR of $17 \%$ in 52 patients with advanced melanoma [25]. Other anti-PDL1 antibodies, atezolizumab and durvalumab, in ongoing phase I trials have also exhibited clinical activities in patients with NSCLC. The RR values of these antibodies and the sample sizes of the respective studies are as follows: atezolizumab, $30 \%(n=43)$ [26]; durvalumab, $13 \%$ $(n=8)$ [27].

\section{Lung cancer}

Representative phase II or III clinical trials with anti-PD-1 antibodies, nivolumab and pembrolizumab, in patients with non-small cell lung cancer (NSCLC) are described here. A phase II single-arm trial of nivolumab in 117 patients with advanced refractory squamous NSCLC yielded an RR of $14.5 \%$; median duration of response had not been reached at the time of analysis. Although the RR was relatively low, there is currently no standard of care in this third-line setting. Nivolumab has also exhibited promising effects as a second-line treatment for squamous NSCLC [28].

In a randomized phase III trial comparing nivolumab $(n=131)$ to docetaxel $(n=129)$ in patients with squamous NSCLC who had disease recurrence after one prior platinum-containing regimen, the median OS was 9.2 months in the nivolumab group versus 6.0 months in the docetaxel group. At 1 year, the overall survival rate was $42 \%$ with nivolumab versus $24 \%$ with docetaxel. The risk of death was $41 \%$ lower with nivolumab than with docetaxel (HR, $0.59 ; P<0.001$ ); the response rate was $20 \%$ with nivolumab versus $9 \%$ with docetaxel $(P=0.008)$, and the median progression-free survival (PFS) was 3.5 months with nivolumab versus 2.8 months with docetaxel (HR, $0.62 ; P<0.001$ ) [29]. Based on these interim data, in 2014 the FDA expanded the approval of nivolumab to include squamous NSCLC with disease recurrence after one prior platinum-containing regimen. 
In a randomized phase III trial comparing nivolumab $(n=292)$ to docetaxel $(n=290)$ in non-squamous NSCLC after failure of platinum-doublet chemotherapy, OS was 12.2 months in the nivolumab group versus 9.4 months in the docetaxel group. At 1 year, the overall survival rate was $51 \%$ with nivolumab versus $39 \%$ with docetaxel [30]. Based on these data, in late 2015 the FDA significantly expanded the approval of nivolumab to previously treated metastatic NSCLC. Additionally, in the first-line setting, a randomized phase III trial is currently underway to compare nivolumab to investigator's choice chemotherapy in patients with previously treated or untreated NSCLC whose tumors express PD-L1 [31].

Four anti-PD-L1 antibodies in the early phase of clinical trials have also demonstrated clinical activity in patients with NSCLC. The RR values of these antibodies and the sample sizes of the respective studies are as follows: BMS936559, $10 \%(n=49)$ [25]; durvalumab, $16 \%(n=58)$ [32]; atezolizumab, $21 \%(n=53)$ [27]; avelumab, $12 \%$ $(n=184)$ [33].

\section{Renal cell carcinoma}

In a phase II clinical trial of nivolumab in 168 patients with metastatic renal cell carcinoma (RCC) patients who had previously received vascular endothelial growth factor (VEGF) pathway inhibitors, RR was $21 \%$ [34]. In a randomized phase III trial of nivolumab in 821 patients with advanced clear cell RCC, the median OS was 25.0 months with nivolumab and 19.6 months with everolimus. RR was higher with nivolumab than with everolimus ( $25 \%$ vs. $5 \%$ ), whereas the median PFS was 4.6 months with nivolumab and 4.4 months with everolimus [35]. Based on these data, in late 2015 the FDA approved nivolumab for RCC patients who had received previous treatment with antiangiogenic therapy in late 2015.

Anti-PD-L1 antibodies in phase I clinical trials for RCC have also demonstrated clinical activity in patients with RCC. The RR values of these antibodies and the sample sizes of the respective studies are as follows: BMS-936559, $12 \%(n=17)$ [25] and atezolizumab, $14 \%(n=56)$ [27] in patients with $\mathrm{RCC}$.

\section{Ovarian cancer}

Based on our clinical studies of cancer immune escape in ovarian cancer [14, 36, 37], we conducted the first principal investigator-initiated two-cohort ( 1 or $3 \mathrm{mg} / \mathrm{kg}, n=10$ each), phase II clinical trial of nivolumab in 20 patients with platinum-resistant recurrent ovarian cancer [38]. The trial was conducted in collaboration with Professor Honjo, who discovered the PD-1 gene [1]. RR at $3 \mathrm{mg} / \mathrm{kg}$ was $20 \%$, including 2 cases of a durable complete response (CR).
Among all 20 patients, RR was $15 \%$ and DCR was $45 \%$; median PFS and OS were 3.5 and 20.0 months, respectively [39]. In our ongoing follow-up study subsequent to this trial, a durable antitumor response with nivolumab has been observed in 2 patients with a CR for more than 1 year [40]. After completing the 1-year nivolumab treatment according to our study design, these 2 patients have each survived without any disease progression for more than 1 year, with no adjuvant antitumor treatment [41].

Later, the interim results became available from two other phase Ib clinical trials with anti-PD-1 antibody [25] and anti-PD-L1 antibody (avelumab). In the trial of pembrolizumab in 26 patients with PD-L1+ advanced ovarian cancer, RR was $11.5 \%$ [42]. In the trial of avelumab in 75 patients with recurrent or refractory ovarian cancer, RR was $10.7 \%$ [43].

\section{Head and neck cancer}

The anti-PD-1 antibody pembrolizumab exhibited antitumor activity in patients with refractory squamous cell carcinoma of the head and neck cancer (SCCHN), whose tumors expressed $\geq 1 \%$ PD-L1 (RR $=20 \%$ in 56 patients) [44]. Anti-PD-L1 antibodies in phase I clinical trials for SCCHN have also exhibited clinical activity. The RR values of these antibodies and the sample sizes of the respective studies are as follows: durvalumab, $14 \%(n=22)$ [45]; atezolizumab, $17 \%(n=6)$ [27].

\section{Bladder cancer}

In a phase I clinical trial of pembrolizumab in 29 patients with advanced bladder cancer patients whose tumors expressed $\geq 1 \%$ PD-L1, RR was $24 \%$, including three cases of CR [46]. Another anti-PD-L1 antibody, atezolizumab, yielded a RR of $26 \%$ in 68 patients with advanced bladder cancer [47]. Based on these findings, in 2014 the FDA set the 'Breakthrough' designation for the clinical development of atezolizumab for treatment of advanced bladder cancer.

\section{Gastric cancer}

In a phase I trial of pembrolizumab in 39 patients with advanced gastric cancer, RR was $22 \%$. Median time to response was 8 weeks, with a median response duration of 24 weeks. The 6-month PFS and OS rates were $24 \%$ and $69 \%$, respectively [48].

\section{Colon cancer}

In a phase II clinical trial of pembrolizumab in 50 patients with colon cancer, RR was higher in the mismatch 
repair-deficient group $(n=25)$ than in the mismatch repairproficient group $(n=25), 62 \%$ versus $0 \%$ [49] (see "Clinical perspectives and issues of PD-1 inhibitors" for details).

\section{Esophageal cancer}

In a phase I study of pembrolizumab in 22 patients with esophageal cancer, RR was $23 \%$, including 3 (11\%) cases of CR [50].

\section{Hepatocellular cancer}

In a phase I/II trial of nivolumab in 42 patients with hepatocellular carcinoma, CR was reported in 2 patients (5\%) and PR in 7 patients $(18 \%)$ for an overall objective RR of $23 \%$. Overall survival (OS) rate at 6 months was $72 \%$ [51].

\section{Breast cancer}

In a phase $1 \mathrm{~b}$ trial of pembrolizumab in 25 patients with advanced triple-negative breast cancer (TNBC) whose tumors expressed $\geq 1 \%$ of PD-L1, RR was $12 \%$ [52]. A phase Ia study of the anti-PD-L1 antibody atezolizumab in 27 patients with metastatic TNBC yielded a RR of $19 \%$, including 1 CR and 4 PRs [53].

\section{Hematological malignancies}

Nivolumab has shown dramatic antitumor effects in patients with relapsed or refractory Hodgkin's lymphoma (HL) $(n=23)$, with a RR of $87 \%$ and a DCR of $100 \%$, including CR in $20 \%$ of patients [54].

Pembrolizumab also yielded a RR of $53 \%$ in 15 patients with pre-treated HL, including CR in $20 \%$ of patients [55]. In a phase I trial of nivolumab in patients with non-HL, diffuse follicular lymphoma $(n=10)$ or large B-cell lymphoma $(n=11)$, RR values were $40 \%$ and $36 \%$, respectively [56].

\section{Clinical perspectives and issues of PD-1 inhibitors}

The accumulated data from clinical trials for solid tumors revealed that the antitumor response rate of PD-1 inhibitors seems not so high. Therefore, there is an urgent need to resolve several issues related to PD-1 inhibitors (Fig. 3). First, to enhance the antitumor effect of PD-1 inhibitors, we have to find the best combination therapy with other antitumor therapy such as chemotherapy, targeted therapy, radiotherapy, or other immunotherapy. Second, because PD-1 inhibitors are very expensive, it is necessary to identify predictive biomarkers that allow selection of appropriate patients. Third, we must learn how to manage severe immunological side effects. Last, we have to investigate the most valuable application of PD-1 inhibitors.

\section{Development of effective combination therapies}

Clinical trials of combination treatments incorporating PD-1 inhibitors with conventional chemotherapies [57], molecular targeted drugs such as PARP inhibitors (olaparib and cediranib) for solid tumors [58], or multi-kinase inhibitor (sunitinib) for RCC [59], focal radiation therapy, and cancer immunomodulators are now underway. In particular, therapy using double-checkpoint inhibitors in combination with nivolumab and ipilimumab for the treatment of advanced melanoma led to longer PFS than either agent alone [60]. However, the frequency of grade 3 or 4 immune-related AEs was also amplified to more than $50 \%$. Similar combinations are now being clinically applied to diverse cancer types, including RCC [61], NSCLC [62], or ovarian cancer [63].

Next-generation clinical trials are also underway for PD-1 inhibitors in combination with other immune modulators such as anti-lymphocyte activation gene 3 (LAG3) antibody for solid tumors [64], anti-killer inhibitory receptor (KIR) antibody, lirilumab, for solid tumors [65], antiOX40 agonistic antibody, MEDI6383, for solid tumors [66], anti-4-1BB agonistic antibody, urelumab, for solid tumors and B-cell non-Hodgkin lymphoma [67], and GMCSF-producing and CD40L-expressing bystander cell line (GM.CD40L) vaccine for NSCLC [68].

\section{Identification of biomarkers}

The identification of predictive biomarkers of PD-1 inhibitors is a crucial next step for advancing the applications of these drugs. Potential predictive biomarkers of antitumor response to PD-1 inhibitors can be considered in either tumor cell-related factors or host immunological factors. Recent studies identified several candidate biomarkers: expression of PD-L1 in tumor cells and tumor-infiltrating lymphocytes, frequencies of mutations in tumor cells, and diversity of tumor antigen-specific $\mathrm{T}$ cells (T-cell repertoire). All these candidates are regarded as viable prospects, although some concerns are associated with each of them.

\section{$P D-L 1$ and TILs}

Since the first clinical trial of nivolumab in 2010 [19], several reports of clinical trials of PD-1 inhibitors have shown that the therapeutic efficacy of PD-1 inhibitors is modestly correlated with PD-L1 expression in tumors [20, 22, 27, 47, $48,69,70]$. In addition, with respect to tumors such as melanoma and bladder cancer, not only tumor cells, but also 
the number of infiltrating $\mathrm{T}$ cells and the proportion of $\mathrm{T}$ cells positive for PD-L1 or PD-1 expression, can be used as indices of therapeutic efficacy [27, 47, 71]. In other words, the effects of PD-1 pathway-inhibiting drugs occur when tumor cells and immune cells are present at a particular locus and the cancer-immunosuppressive state mediated by the PD-1 pathway has been established. In future, in addition to follow-up studies, diagnostic-grade evaluation and accuracy control of PD-L1 expression will also be required. Therefore, there is a need to establish clearer and more accurate analysis methods [72].

On the other hand, it is probable that PD-L1 expression by tumors will change during treatment in response to the immune state and the administration of therapeutic drugs [27, 73-75]. Therefore, it is necessary to investigate the influence of parameters such as the timing and locus of sample collection by biopsies and surgery; whether samples are embedded in paraffin or frozen; and evaluation methods such as immunostaining, quantitative polymerase chain reaction (PCR), and Western blotting. In addition, in cases of tumors within an abdominal cavity, whether primary, secondary, or metastatic, it is often difficult to carry out biopsies from the body surface. Consequently, various issues must be investigated and analyzed, such as the different sampling modes needed to collect different types of tumor tissue.

Furthermore, since in the case of nivolumab for Hodgkin's lymphoma patients, the response rate was $87 \%$ and the disease-control rate was $100 \%$ [54], biomarkers per se are not useful in such a type of tumor for which efficacy has been demonstrated. On the other hand, in a phase I/II nivolumab study in SCLC patients [76], a phase III nivolumab study in lung squamous cell carcinoma patients [29], and a phase III study of nivolumab plus ipilimumab combination therapy in melanoma patients [60], no significant correlations were observed between PD-L1 expression and therapeutic efficacy. However, in the nivolumab plus ipilimumab combination therapy study, a subanalysis did reveal correlations between PD-L1 expression and therapeutic efficacy in the nivolumab monotherapy group. Therefore, it might be useful to establish a paradigm for therapy selection in which nivolumab monotherapy is used when a tumor is positive for PD-L1 expression, but combination therapy is used when it is negative. In all these studies, therapeutic efficacy was observed even with PD-L1-negative patients, and it is therefore probably inappropriate to select patients solely on the basis of whether they express PD-L1 [60]. Thus, there is a demand for immunologically individualized treatment methods that can be selected accurately, with consideration given to other factors such as cancer type and histological subtype.

\section{Relationship between PD-1 signal and genomic mutation}

Rapid progress has also been made in reverse-translational research using specimens from patients who received immune checkpoint inhibitors. In addition, recent progress in genomic analysis using next-generation sequencing techniques has enabled comprehensive detection of mutations in cancer tissue. According to The Cancer Genome Atlas, which covers 7042 tumor samples from 30 cancer types, the frequency of somatic gene mutations is highest in melanoma, followed by lung squamous cell carcinoma, lung adenocarcinoma, bladder cancer, stomach cancer, and esophageal adenocarcinoma [77]. PD-1-inhibitors are expected to be therapeutically effective in cancer types with high somatic gene mutation frequencies $[78,79]$. In other words, cancer types with numerous mutations necessarily also express numerous mutant cancer antigens, and immunosuppression mediated by the PD-1 pathway occurs in the presence of numerous $T$ cells that specifically recognize those mutant antigens. Thus, PD-1 pathway inhibitors represent a promising class of drugs for use in such cases (Fig. 2). This position supports the findings of fundamental research using a mouse model that demonstrated the existence of mutant cancer antigen-specific $\mathrm{T}$ cells, as well as reactivation of these cells by anti-PD-1 antibodies and antiCTLA-4 antibodies (CTLA-4 is a receptor for the same immunosuppressive signals as PD-1) [80].

Snyder et al. performed whole-exome sequencing of tumors from 64 melanoma patients who had been treated with the anti-CTLA-4 antibody ipilimumab or tremelimumab. The results revealed durable clinical efficacy in 11 subjects, and the mutation levels in these patients were significantly elevated [81]. Because neither of these factors is sufficient as a predictive marker for treatment, genomewide somatic cell neo-epitope analysis and HLA analysis were carried out, resulting in identification of a neo-epitope candidate that is specifically expressed in tumors against which anti-CTLA-4 antibodies are therapeutically effective. This neo-epitope was validated in a dataset comprising 39 melanoma patients. In addition, the neo-epitope activated $\mathrm{T}$ cells derived from patients who received ipilimumab, demonstrating the usefulness of mutation analysis by whole-exome sequencing, as well as neo-epitope analysis, in predicting the therapeutic efficacy of anti-CTLA-4 antibodies.

In addition, Rizvi et al. carried out whole-exome sequence analysis of tumors in NSCLC patients treated with the anti-PD-1 antibody pembrolizumab. The results revealed that when numerous non-synonymous mutations were present, there were correlations between response to treatment, durable clinical benefit (i.e., partial response or stable disease for at least 6 months), and recurrence-free 


\section{Cancer cell}
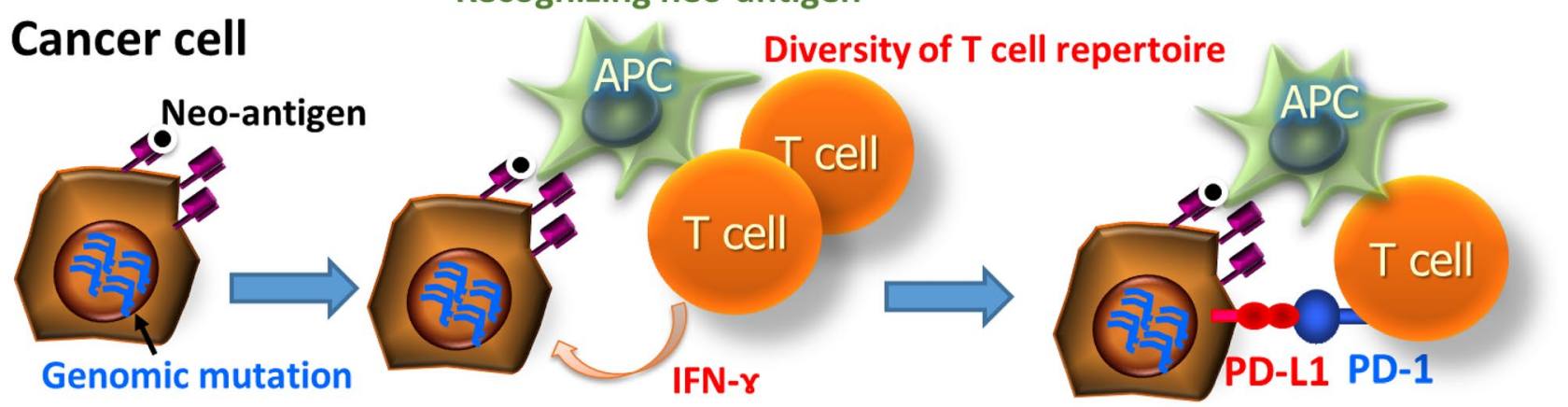

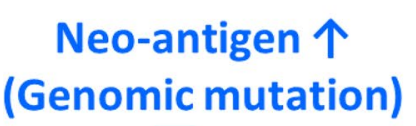

Mutanome

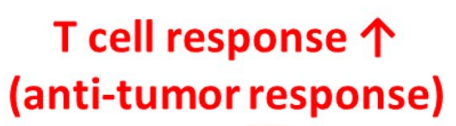

(anti-tumor response)
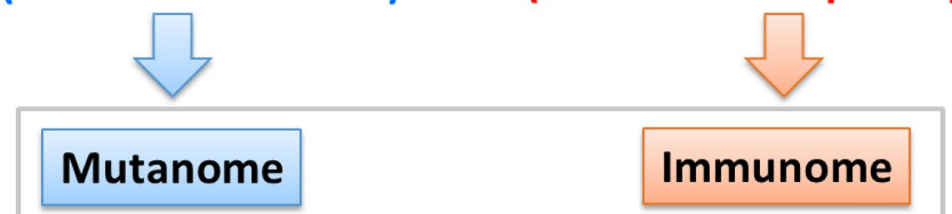

Immunome

\section{Recognizing neo-antigen}

\section{Predictive biomarker of PD-1/PD-L1 inhibitors?}

Fig. 2 Relationship between PD-1 signal and genomic mutation. Mutated neo-antigens are expressed on the surface of a cancer cell in response to genomic mutation and amplification of a cancer cell. Recognition of a neo-antigen as a foreign body by an antigen-presenting cell (APC) induces a T-cell response, and consequently the activated $\mathrm{T}$ cell releases interferon (IFN)- $\gamma$. A cancer cell that is exposed to IFN- $\gamma$ expresses PD-L1, thereby establishing an acquired immune

survival rate [82]. Similarly, correlations were observed between therapeutic efficacy and a set of genes that is upregulated in smokers, neo-antigen count, and mutations in the DNA-repair pathway, all of which are linked to the mutation level. Furthermore, some studies have described patients who exhibit neo-antigen-specific T-cell immune responses that increase with tumor contraction upon treatment with pembrolizumab. Therefore, it is possible that the efficacy of pembrolizumab treatment against lung cancer is determined by the genomic landscape of the cancer.

In addition, Le et al. found that in a phase II tremelimumab study carried out previously in CRC patients, 1 of 47 subjects exhibited a partial response. In addition, in a phase I study in which the anti-PD-L1 antibody MPDL3280A was administered to 20 subjects, 1 CRC patient with deletion of a mismatch repair (MMR) gene exhibited a partial response [49]. Therefore, the antiPD-1 antibody pembrolizumab was administered to three cohorts, A, B, and C, respectively, comprising 25 CRC patients with MMR deletion, 25 CRC patients with normal MMR, and 21 patients with cancers other than CRC with MMR deletion. The therapeutic efficacy was very high in the CRC patients with MMR deletion, with a response rate of $62 \%$ and a disease-control rate of $92 \%$. By contrast, in the 25 CRC patients with normal MMR, the efficacy was resistance. In this type of tumor microenvironment, a PD-1 pathway inhibitor should be effective; thus, genome-wide mutation analysis (i.e., mutanome analysis) of cancer cells using next-generation sequencing technology and diversity analysis of the T-cell repertoire (i.e., the immunome) have attracted attention as strategies for identification of predictive biomarkers. APC antigen-presenting cell

very low, with a response rate of $0 \%$ and a disease-control rate of $16 \%$. Furthermore, in the subjects with nonCRC cancers with MMR deletions, the response rate and disease-control rate were $60 \%$ and $70 \%$, respectively, suggesting the possibility that MMR deletion is a predictive factor for the therapeutic efficacy of anti-PD-1 antibody, pembrolizumab.

In the manner already described, a search for biomarkers was recently carried out via comprehensive mutation analysis of the cancer genome using next-generation sequencing technology. This approach is termed mutanome analysis when it involves genome-wide mutation analysis of cancer cells, and immunome analysis when it involves a comprehensive exploratory analysis related to tumor immunology; the latter includes diversity analysis of a T-cell repertoire, microarray analysis, and protein analysis. By making extensive use of these techniques, high-throughput extraction of markers can be carried out more effectively, and rapid progress is being made in verification techniques for validation studies even in the field of cancer immunology (Fig. 2).

Integrating several biomarkers in an algorithm

As already mentioned, several candidates for predictive biomarkers were recently identified. Further study is 
COMBINATION?

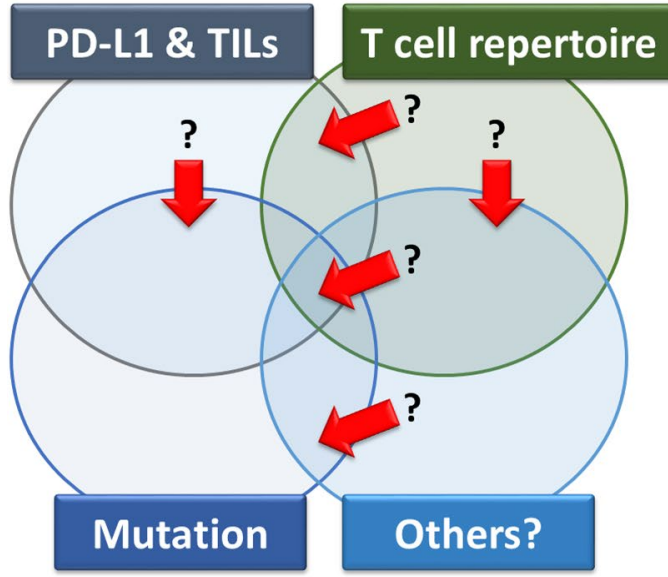

ALGORITHM?

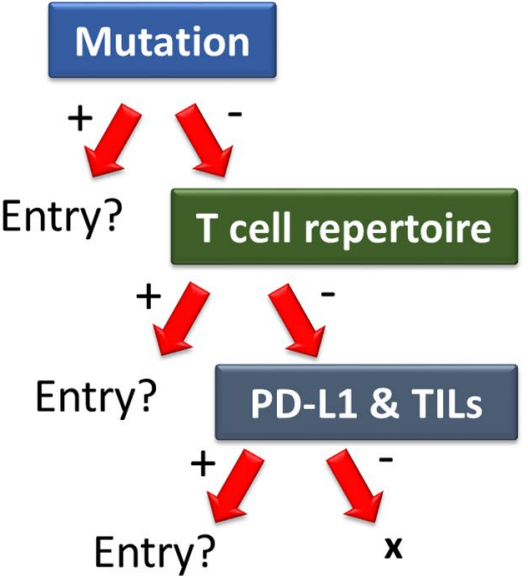

Fig. 3 Identification of the best biomarker strategy. A combination or algorithm of biomarkers may be needed. For example, it is important to understand whether it will be necessary to use a combination of biomarkers, e.g., PD-L1+ tumor cells, PD-L1+, and/or PD-1+ tumor-infiltrating lymphocytes, frequencies of neo-antigens and tumor mutations, and diversity of the T-cell repertoire. Additionally, more candidate predictive biomarkers will be identified in the future. Therefore, development of an algorithm or combination of these biomarkers should be a high priority for future work

motor sequelae, myocarditis and cardiac insufficiency, and acute adrenal insufficiency and nephritis [83, 84]. In the first phase II trial of nivolumab, grade 1 or 2 pneumonitis in 6 of 296 patients was reversible upon discontinuation of treatment and administration of glucocorticoid, but 3 of 296 patients $(1 \%)$ died of pneumonitis. On the basis of these findings, guidelines and specific algorithms for identification, early intervention, and management of irAEs have been developed $[20,25]$. In the clinical setting, if severe irAEs occur, clinicians should perform a timely evaluation to confirm the diagnosis, and if necessary, admit the patient to the hospital and treat with intravenous corticosteroids without hesitation. Moreover, if corticosteroids are not effective, clinicians should consider using additional immunosuppressive drugs such as anti-tumor necrosis factor (TNF) antibody (i.e., infliximab) [83].

Although irAEs can develop at any time, the majority of immune toxicities of nivolumab occur within the first 4 months $[84,85]$. The median time to onset of irAEs tends to differ depending on the type of toxicity, and can be roughly classified as early ( $<2$ months) or late ( $>2$ months). Early toxicities include skin (5 weeks), gastrointestinal (7 weeks), and hepatic (7 weeks), whereas late toxicities include pulmonary (9 weeks), endocrine (10 weeks), and renal (15 weeks). However, clinicians should keep in mind that all types of irAEs can occur at any time [84, 87-89].

Finally, we, as gynecologists, believe that we should investigate the gender differences in frequency and severity of side effects and evaluate the risk of infertility for younger men or women who receive PD-1 inhibitors.

risk after corticosteroid therapy, encephalopathy and neu- 


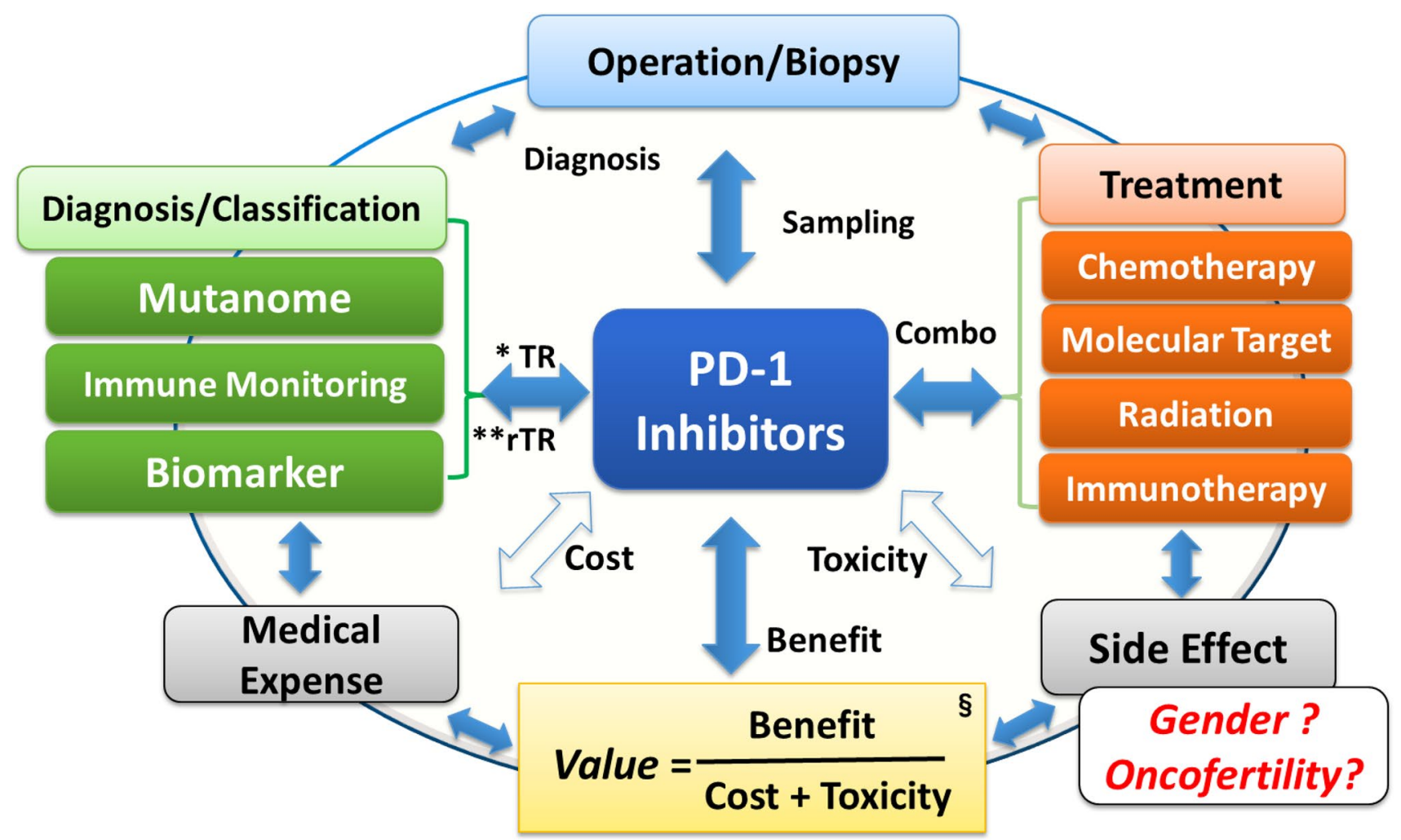

Fig. 4 Perspectives and issues regarding PD-1 inhibitors. Asterisk TR translational research, double asterisk rTR reverse translational research. Section sign See reference [89]

Currently, very little is known about the influence of PD-1 inhibitors on human fertility or pregnancy (Fig. 3). The drug package insert of nivolumab [86] describes embryofetal toxicity in the cynomolgus monkey and advises females with reproductive potential to use effective contraception during treatment with nivolumab and for at least 5 months after the last dose.

\section{The value of PD-1 inhibitors}

On the other hand, the American Society of Clinical Oncology increasingly emphasizes the value of anticancer treatments, referring to their benefit/cost ratio [90, 91]. In other words, in future, for anti-cancer treatments to be considered excellent, evaluation of the cost (i.e., medical expenses) and toxicity (i.e., AEs) of each will be recommended, in addition to its benefit (antitumor efficacy); furthermore, it will be important to select overall treatments that are appropriate for the individual patient's background and physical condition (Fig. 4). In this context, to judge the value of PD-1 pathway inhibitors, there is an urgent need to establish patient selection methods based on biomarkers, permitting prediction of the efficacy and AEs of PD-1 pathway inhibitors.

\section{Conclusion}

More than 20 years after the discovery of PD-1 [1], several studies have identified the clinical efficacy of PD-1 blockade against a wide spectrum of solid and hematological malignancies, opening the door to a strategy for the treatment of cancer. In addition, on the basis of reports of clinical findings obtained using PD-1 inhibitors, longstanding theories regarding cancer immune surveillance [92] and cancer immunoediting, including the mechanism of cancer immune escape [93, 94], have recently been confirmed by fundamental research on tumor immunology. Nevertheless, a great deal of fundamental, exploratory research remains to be done on areas such as predictive biomarkers for therapeutic efficacy and adverse drug reactions. In future, we anticipate that clinical studies of PD-1 inhibitors and deep reverse-translational programs involving molecular and genomic research will reveal fundamental details of PD-1/ PD-L1 signaling.

Acknowledgments We deeply thank Professors Tasuku Honjo, Taku Okazaki, and Shunsuke Chikuma at the Department of Immunology and Genomic Medicine in Kyoto University and Dr. Shingo Fujii at Kitano Hospital. This work was supported by the Project for Development of Innovative Research on Cancer Therapeutics 
(P-DIRECT)/Ministry of Education, Culture, Sports, Science and Technology.

\section{Compliance with ethical standards}

Conflict of interest Hamanishi and Matsumura received a research grant from Daiichisankyo; Konishi received honoraria for KanaharaShuppan Co, and Medicalview Co.; Mandai, Baba, and Abiko have no conflict of interest.

Open Access This article is distributed under the terms of the Creative Commons Attribution 4.0 International License (http://creativecommons.org/licenses/by/4.0/), which permits unrestricted use, distribution, and reproduction in any medium, provided you give appropriate credit to the original author(s) and the source, provide a link to the Creative Commons license, and indicate if changes were made.

\section{References}

1. Dunn GP, Bruce AT, Ikeda $\mathrm{H}$ et al (2002) Cancer immunoediting: from immunosurveillance to tumor escape. Nat Immunol 11:991-998

2. Ishida Y, Agata Y, Shibahara K et al (1992) Induced expression of PD-1, a novel member of the immunoglobulin gene superfamily, upon programmed cell death. EMBO J 11:3887-3895

3. Iwai Y, Ishida M, Tanaka Y et al (2002) Involvement of PD-L1 on tumor cells in the escape from host immune system and tumor immunotherapy by PD-L1 blockade. Proc Natl Acad Sci USA 99:12293-12297

4. Iwai Y, Terawaki S, Honjo T (2005) PD-1 blockade inhibits hematogenous spread of poorly immunogenic tumor cells by enhanced recruitment of effector $\mathrm{T}$ cells. Int Immunol 17:133-144

5. Hirano F, Kaneko K, Tamura H et al (2005) Blockade of B7-H1 and PD-1 by monoclonal antibodies potentiates cancer therapeutic immunity. Cancer Res 65:1089-1096

6. Freeman GJ, Long AJ, Iwai Y et al (2000) Engagement of the PD-1 immunoinhibitory receptor by a novel B7 family member leads to negative regulation of lymphocyte activation. J Exp Med 192:1027-1034

7. Okazaki T, Honjo T (2007) PD-1 and PD-1 ligands: from discovery to clinical application. Int Immunol 19:813-824

8. Dong H, Strome SE, Salomao DR et al (2002) Tumor-associated B7-H1 promotes T-cell apoptosis: a potential mechanism of immune evasion. Nat Med 8:793-800

9. Keir ME, Butte MJ, Freeman GJ et al (2008) PD-1 and its ligands in tolerance and immunity. Annu Rev Immunol 26:677-704

10. Blank C, Brown I, Peterson AC et al (2004) PD-L1/B7H-1 inhibits the effector phase of tumor rejection by $\mathrm{T}$ cell receptor (TCR) transgenic CD8+ T cells. Cancer Res 64:1140-1145

11. Curiel TJ, Wei S, Dong H et al (2003) Blockade of B7-H1 improves myeloid dendritic cell-mediated antitumor immunity. Nat Med 9:562-567

12. Thompson RH, Gillett MD, Cheville JC et al (2004) Costimulatory B7-H1 in renal cell carcinoma patients: indicator of tumor aggressiveness and potential therapeutic target. Proc Natl Acad Sci USA 101:17174-17179

13. Ohigashi Y, Sho M, Yamada Y et al (2005) Clinical significance of programmed death-1 ligand-1 and programmed death-1 ligand-2 expression in human esophageal cancer. Clin Cancer Res 11:2947-2953
14. Hamanishi J, Mandai M, Iwasaki M et al (2007) Programmed cell death 1 ligand 1 and tumor-infiltrating CD8+ T lymphocytes are prognostic factors of human ovarian cancer. Proc Natl Acad Sci USA 104:3360-3365

15. Nomi T, Sho M, Akahori T et al (2007) Clinical significance and therapeutic potential of the programmed death-1 ligand/ programmed death-1 pathway in human pancreatic cancer. Clin Cancer Res 13:2151-2157

16. Konishi J, Yamazaki K, Azuma M et al (2004) B7-H1 expression on non-small cell lung cancer cells and its relationship with tumor-infiltrating lymphocytes and their PD-1 expression. Clin Cancer Res 10:5094-5100

17. Wu C, Zhu Y, Jiang J et al (2006) Immunohistochemical localization of programmed death-1 ligand-1 (PD-L1) in gastric carcinoma and its clinical significance. Acta Histochem 108:19-24

18. Ghebeh H, Mohammed S, Al-Omair A et al (2006) The B7-H1 (PD-L1) T lymphocyte-inhibitory molecule is expressed in breast cancer patients with infiltrating ductal carcinoma: correlation with important high-risk prognostic factors. Neoplasia 8:190-198

19. Brahmer JR, Drake CG, Wollner I et al (2010) Phase I study of single-agent anti-programmed death-1 (MDX-1106) in refractory solid tumors: safety, clinical activity, pharmacodynamics, and immunologic correlates. J Clin Oncol 28:3167-3175

20. Topalian SL, Hodi FS, Brahmer JR et al (2012) Safety, activity, and immune correlates of anti-PD-1 antibody in cancer. N Engl J Med 366:2443-2454

21. Topalian SL, Sznol M, McDermott DF et al (2014) Survival, durable tumor remission, and long-term safety in patients with advanced melanoma receiving nivolumab. J Clin Oncol 32:1020-1030

22. Robert C, Long GV, Brady B et al (2015) Nivolumab in previously untreated melanoma without BRAF mutation. N Engl J Med 372:320-330

23. Weber JS, D’Angelo SP, Minor D et al (2015) Nivolumab versus chemotherapy in patients with advanced melanoma who progressed after anti-CTLA-4 treatment (CheckMate 037): a randomised, controlled, open-label, phase 3 trial. Lancet Oncol $16: 375-384$

24. Robert C, Schachter J, Long GV et al (2015) Pembrolizumab versus ipilimumab in advanced melanoma. $\mathrm{N}$ Engl $\mathrm{J}$ Med 372:2521-2532

25. Brahmer JR, Tykodi SS, Chow LQ et al (2012) Safety and activity of anti-PD-L1 antibody in patients with advanced cancer. N Engl J Med 366:2455-2465

26. Lutzky J, Antonia SJ, Blake-Haskins A et al. (2014) A phase 1 study of MEDI4736, an anti-PD-L1 antibody, in patients with advanced solid tumors. 2014 ASCO Annual Meeting. J Clin Oncol 32:5s (suppl: abstr 3001)

27. Herbst RS, Soria JC, Kowanetz M et al (2014) Predictive correlates of response to the anti-PD-L1 antibody MPDL3280A in cancer patients. Nature (Lond) 515:563-567

28. Rizvi NA, Mazières J, Planchard D et al (2015) Activity and safety of nivolumab, an anti-PD-1 immune checkpoint inhibitor, for patients with advanced, refractory squamous non-small-cell lung cancer (CheckMate 063): a phase 2, single-arm trial. Lancet Oncol 16:257-265

29. Brahmer J, Reckamp KL, Baas P et al (2015) Nivolumab versus docetaxel in advanced squamous-cell non-small-cell lung cancer. N Engl J Med 373:123-135

30. Borghaei H, Paz-Ares L, Horn L et al (2015) Nivolumab versus docetaxel in advanced nonsquamous non-small-cell lung cancer. N Engl J Med 373:1627-1639

31. Clinical Trials. gov. NCT02041533 
32. Brahmer JR, Rizvi NA, Lutzky J et al. (2014) Clinical activity and biomarkers of MEDI4736, an anti-PD-L1 antibody, in patients with NSCLC. 2014 ASCO Annual Meeting. J Clin Oncol 32:5s (suppl: abstr 8021)

33. Gulley JL, Spigel D, Kelly K et al. (2015) Avelumab (MSB0010718C), an anti-PD-L1 antibody, in advanced NSCLC patients: a phase $1 \mathrm{~b}$, open-label expansion trial in patients progressing after platinum-based chemotherapy. 2015 ASCO Annual Meeting. J Clin Oncol 33 (suppl: abstr 8034)

34. Motzer RJ, Rini BI, McDermott DF et al (2015) Nivolumab for metastatic renal cell carcinoma: results of a randomized phase II trial. J Clin Oncol 33:1430-1437

35. Motzer RJ, Escudier B, McDermott DF et al (2015) Nivolumab versus everolimus in advanced renal-cell carcinoma. $\mathrm{N}$ Engl $\mathrm{J}$ Med 373:1803-1813

36. Hamanishi J, Mandai M, Matsumura N et al (2010) Activated local immunity by CC chemokine ligand 19-transduced embryonic endothelial progenitor cells suppresses metastasis of murine ovarian cancer. Stem Cells 28:164-173

37. Hamanishi J, Mandai M, Abiko K et al (2011) The comprehensive assessment of local immune status of ovarian cancer by the clustering of multiple immune factors. Clin Immunol 141:338-347

38. Hamanishi J, Mandai M, Ikeda T et al. (2014) Efficacy and safety of anti-PD-1 antibody (Nivolumab: BMS-936558, ONO4538) in patients with platinum-resistant ovarian cancer. 2014 ASCO Annual Meeting, Clinical Science Symposium. J Clin Oncol 32:5s (suppl: abstr 5511)

39. Hamanishi J, Mandai M, Ikeda T et al (2015) Efficacy and safety of anti-PD-1 antibody (Nivolumab: BMS-936558, ONO-4538) in patients with platinum-resistant ovarian cancer. J Clin Oncol 33:4015-4022

40. Hamanishi J, Mandai M, Ikeda T, et al. (2015) Durable tumor remission in patients with platinum-resistant ovarian cancer receiving nivolumab. 2015 ASCO Annual Meeting. J Clin Oncol 33 (suppl: abstr 5570)

41. Hamanishi J (2015) Immunotherapy for gynaecological cancers: Challenges and opportunities. ESMO Asia 2015. Special Symposium. 18 December 2015, Singapore

42. Varga A, Piha-Paul SA, Ott PA et al. (2015) Antitumor activity and safety of pembrolizumab in patients (pts) with PD-L1 positive advanced ovarian cancer: interim results from a phase Ib study. 2015 ASCO Annual Meeting. J Clin Oncol 33 (suppl: abstr 5510)

43. Disis ML, Patel MR, Pant $S$ et al. (2015) Avelumab (MSB0010718C), an anti-PD-L1 antibody, in patients with previously treated, recurrent or refractory ovarian cancer: a phase Ib, open-label expansion trial. 2015 ASCO Annual Meeting. J Clin Oncol 33 (suppl: abstr 5509)

44. Seiwert TY, Burtness B, Weiss J et al. (2014) A phase Ib study of MK-3475 in patients with human papilloma virus (HPV)-associated and non-HPV-associated head and neck $(\mathrm{H} / \mathrm{N})$ cancer. 2014 ASCO Annual Meeting. J Clin Oncol 32:5s (suppl: abstr 6011)

45. Segal NH, Antonia SJ, Brahmer JR, et al. (2014) Preliminary data from a multi-arm expansion study of MEDI4736, an antiPD-L1 antibody. 2014 ASCO Annual Meeting. J Clin Oncol 32:5s (suppl: abstr 3002)

46. Plimack ER, Gupta S, Bellmunt J et al. (2014) Phase 1B study of pembrolizumab (pembro; MK-3475) in patients (pts) with advanced urothelial tract cancer. Ann Oncol (Meeting Abstracts) 25:LBA23A

47. Powles T, Eder JP, Fine GD et al (2014) MPDL3280A (anti-PDL1) treatment leads to clinical activity in metastatic bladder cancer. Nature (Lond) 515:558-562

48. Bang YJ, Chung HC, Shankaran V, et al. (2015) Relationship between PD-L1 expression and clinical outcomes in patients with advanced gastric cancer treated with the anti-PD-1 monoclonal antibody pembrolizumab (MK-3475) in KEYNOTE-012. 2015 ASCO Annual Meeting. J Clin Oncol 33 (suppl: abstr 4001)

49. Le DT, Uram JN, Wang H et al (2015) PD-1 blockade in tumors with mismatch-repair deficiency. N Engl J Med 372:2509-2520

50. Doi T, Piha-Paul SA, Jalal SI, et al. (2015) Pembrolizumab (MK3475) for patients (pts) with advanced esophageal carcinoma. Preliminary results from KEYNOTE-028. 2015 ASCO Annual Meeting. J Clin Oncol 33 (suppl: abstr 4010)

51. El-Khoueiry AB, Melero I, Crocenzi TS et al. (2015) Phase I/ II safety and antitumor activity of nivolumab in patients with advanced hepatocellular carcinoma (HCC): CA209-040. 2015 ASCO Annual Meeting. J Clin Oncol 33 (suppl: abstr LBA101)

52. Nanda R, Chow L, Dees E, et al. (2014) A phase Ib study of pembrolizumab (MK-3475) in patients with advanced triplenegative breast cancer. San Antonio Breast Cancer Symposium, 2014 December 9-13, San Antonio, TX

53. Emens L, Braiteh F, Cassier P et al. (2014) Inhibition of PD-L1 by MPDL3280A leads to clinical activity in patients with metastatic triple-negative breast cancer. 2014 San Antonio Breast Cancer Symposium, 2014 December 9-13, San Antonio, TX

54. Ansell SM, Lesokhin AM, Borrello I et al (2015) PD-1 blockade with nivolumab in relapsed or refractory Hodgkin's lymphoma. N Engl J Med 372:311-319

55. Moskowitz C, Ribrag V, Michot J-M, et al. (2014) PD-1 Blockade with the monoclonal antibody pembrolizumab (MK-3475) in patients with classical Hodgkin lymphoma after brentuximab vedotin failure: preliminary results from a phase $1 \mathrm{~b}$ study (KEYNOTE-013). 56th Annual Meeting of the American Society of Hematology, December 6-9, 2014 (abstr 290)

56. Lesokhin AM, Ansell SM, Armand P et al. (2014) Preliminary results of a phase I study of nivolumab (BMS-936558) in patients with relapsed or refractory lymphoid malignancies. 56th Annual Meeting of the American Society of Hematology, December 6-9, 2014 (abstr 291)

57. Chinai JM, Janakiram M, Chen F et al (2015) New immunotherapies targeting the PD-1 pathway. Trends Pharmacol Sci 36:587-595

58. Clinical Trials. gov. NCT02484404

59. Clinical Trials. gov. NCT02599779

60. Larkin J, Chiarion-Sileni V, Gonzalez R et al (2015) Combined nivolumab and ipilimumab or monotherapy in untreated melanoma. N Engl J Med 373:23-34

61. Hammers HJ, Plimack ER, Infante JR, et al. (2015) Phase I study of nivolumab in combination with ipilimumab in metastatic renal cell carcinoma (mRCC). 2015 ASCO Annual Meeting. J Clin Oncol 32 (suppl: abstr 4504)

62. Patnaik A, Socinski MA, Gubens MA et al.(2015) Phase 1 study of pembrolizumab (pembro; MK-3475) plus ipilimumab (IPI) as second-line therapy for advanced non-small cell lung cancer (NSCLC): KEYNOTE-021 cohort D. 2015 ASCO Annual Meeting. J Clin Oncol 33 (suppl: abstr 8011)

63. Clinical Trials. gov. NCT02498600

64. Clinical Trials. gov. NCT01968109

65. Clinical Trials. gov. NCT01714739

66. Clinical Trials. gov. NCT02221960

67. Clinical Trials. gov. NCT02253992

68. Clinical Trials. gNCT02466568

69. Callahan M. (2015) Understanding the biology behind responses to immunotherapy. 2014 ASCO Annual Meeting. Developmental therapeutics-immunotherapy (Oral Abstract Session as discussant)

70. Lipson EJ, Forde PM, Hammers HJ et al (2015) Antagonists of PD-1 and PD-L1 in cancer treatment. Antagonists of PD-1 and PD-L1 in cancer treatment. Semin Oncol 42:587-600 
71. Taube JM, Anders RA, Young GD et al (2012) Colocalization of inflammatory response with B7-h1 expression in human melanocytic lesions supports an adaptive resistance mechanism of immune escape. Sci Transl Med 4:127-137

72. Garber K (2015) Predictive biomarkers for checkpoints, first tests approved. Nat Biotechnol 33:1217-1218

73. Peng J, Hamanishi J, Matsumura N et al (2015) Chemotherapy induces programmed cell death-ligand 1 overexpression via the nuclear factor- $\kappa \mathrm{B}$ to foster an immunosuppressive tumor microenvironment in ovarian cancer. Cancer Res 75:5034-5045

74. Abiko K, Mandai M, Hamanishi J et al (2013) PD-L1 on tumor cells is induced in ascites and promotes peritoneal dissemination of ovarian cancer through CTL dysfunction. Clin Cancer Res 16:1363-1374

75. Chen L, Han X (2015) Anti-PD-1/PD-L1 therapy of human cancer: past, present, and future. J Clin Invest 125:3384-3391

76. Antonia SJ, Bendell JC, Taylor MH, et al. (2015) Phase I/II study of nivolumab with or without ipilimumab for treatment of recurrent small cell lung cancer (SCLC): CA209-032. 2015 ASCO Annual Meeting. J Clin Oncol 33 (suppl: abstr 7503)

77. Alexandrov LB, Nik-Zainal S, Wedge DC et al (2013) Signatures of mutational processes in human cancer. Nature (Lond) 500:415-421

78. Pardoll M (2015) The ABCs of cancer immunotherapy. 2015 ASCO Annual Meeting, Education Session

79. Postow MA, Callahan MK, Wolchok JD (2015) Immune checkpoint blockade in cancer therapy. J Clin Oncol 33:1974-1982

80. Gubin MM, Zhang X, Schuster H et al (2014) Checkpoint blockade cancer immunotherapy targets tumour-specific mutant antigens. Nature (Lond) 515:577-581

81. Snyder A, Makarov V, Merghoub T et al (2014) Genetic basis for clinical response to CTLA-4 blockade in melanoma. N Engl J Med 371:2189-2199

82. Rizvi NA, Hellmann MD, Snyder KA et al (2015) Mutational landscape determines sensitivity to PD-1 blockade in non-small cell lung cancer. Science. 348:124-128
83. Naidoo J, Page DB, Li BT et al (2015) Toxicities of the antiPD-1 and anti-PD-L1 immune checkpoint antibodies. Ann Oncol 26:2375-2391

84. Champiat S, Lambotte O, Barreau E, et al. (2015) Management of Immune Checkpoint Blockade Dysimmune Toxicities: a collaborative position paper. Ann Oncol. pii: mdv623 [Epub ahead of print]

85. Weber JS, Antonia SJ, Topalian SL, et al. (2015) Safety profile of nivolumab (NIVO) in patients (pts) with advanced melanoma (MEL): a pooled analysis. 2015 ASCO Annual Meeting. J Clin Oncol 33 (suppl: abstr 9018)

86. Highlights of prescribing information of nivolumab http://www. accessdata.fda.gov/drugsatfda_docs/label/2014/125554lbl.pdf

87. Highlights of prescribing information of pembrolizumab. http://www. accessdata.fda.gov/drugsatfda_docs/label/2015/125514s004s006lbl. pdf

88. Postow MA (2015) Managing immunotherapy-related side effects. 2015 ASCO Annual Meeting. The ABCs of Cancer Immunotherapy

89. Sanlorenzo M, Vujic I, Daud A et al (2015) Pembrolizumab cutaneous adverse events and their association with disease progression. JAMA Dermatol 151:1206-1212

90. Saltz L (2015) Perspectives on Value. 2015 ASCO Annual Meeting, Plenary Session Including the Science of Oncology Award and Lecture

91. Schnipper LE, Davidson NE, Wollins DS et al (2015) American Society of Clinical Oncology Statement: a conceptual framework to assess the value of cancer treatment options. J Clin Oncol 33:2563-2577

92. Burnet FM (1967) Immunological aspects of malignant disease. Lancet 1:1171-1174

93. Dunn GP, Bruce AT, Ikeda $\mathrm{H}$ et al (2002) Cancer immunoediting: from immunosurveillance to tumor escape. Nat Immunol 3:991-998

94. Schreiber RD, Old LJ, Smyth MJ (2011) Cancer immunoediting: integrating immunity's roles in cancer suppression and promotion. Science 331:1565-1570 